\title{
Measurement of Stark widths in single ionized oxygen
}

\author{
J.A. del Val ${ }^{\star}$, J.A. Aparicio, V. González, and S. Mar \\ Departamento de Optica, Universidad de Valladolid, 47071 Valladolid, Spain
}

Received November 25, 1998; accepted March 23, 1999

\begin{abstract}
Stark widths of 32 OII spectral lines have been measured, for the first time in 16 cases to the authors' knowledge. A pulsed arc was used as a plasma source. The electron density, determined both interferometrically and spectroscopically, covers the range $(2.5-7.5) 10^{22} \mathrm{~m}^{-3}$. The electron temperature, determined from Boltzmannplots, covers the range of $25000-47000 \mathrm{~K}$. Possible dependencies of Stark width on the temperature have been checked. The measured widths are compared both with theoretical and experimental data available in the literature.
\end{abstract}

Key words: atomic data — line: profile — methods: laboratory

\section{Introduction}

The Stark widths which are measured in accessible and well-known light sources, besides to improve the knowledge about spectral line broadening phenomena, allow to diagnose remote light sources of great interest to astrophysics.

Oxygen plays an important role in astrophysical, technical and laboratory plasmas; nevertheless the amount of available experimental information on the Stark broadening for the species OII continues to be rather scarce. On the other hand, the different theoretical and semiempirical approaches show some discrepancies and need to be compared with experimental measurements.

This work furnishes experimental Stark widths of 32 different OII spectral lines, in the range $400-470 \mathrm{~nm}$, belonging to $3 \mathrm{~s}-3 \mathrm{p}, 3 \mathrm{~s}^{\prime}-3 \mathrm{p}^{\prime}, 3 \mathrm{p}-3 \mathrm{~d}, 3 \mathrm{p}^{\prime}-3 \mathrm{~d}^{\prime}, 3 \mathrm{p}^{\prime \prime \prime}-3 \mathrm{~d}^{\prime \prime \prime}$ and $3 \mathrm{~d}-4 \mathrm{f}$ transition arrays. For 16 of these lines no previous measured data have been found in the literature. The

Send offprint requests to: J.A. del Val

* Permanent address: Departamento de Física Aplicada, Universidad de Salamanca, E. Politécnica Superior, 05071 Avila, Spain.
Stark widths have been measured in relatively large ranges of electron temperature and density. Standard diagnostic techniques, carefully checked, have been used to get these plasma parameters. Double and independent methods have been applied for each parameter, Starting with one wavelength interferometry using the $457.9 \mathrm{~nm}$ and also $514.0 \mathrm{~nm}$ transitions of an argon ion laser and $\mathrm{H}_{\alpha}$ Stark broadening to determine electron density. Second, the intensities of 14 OII selected lines, and also 13 NeII lines emitted by the same plasma, help determine the electron temperature by means of different Boltzmann-plots. The measured profiles have been corrected for Doppler and instrumental broadening. Self-absorption has been proved to be completely negligible.

In the ranges of electron density and temperature of this work the measured Stark widths verify a good linear dependence on plasma electron density. After taking into account the experimental uncertainties, this work results are in good agreement with previous measures like those of Platiša et al. (1975); Djeniže et al. (1991, 1998) and with theoretical works by Griem (1974); Hey \& Breger (1980) and Dimitrijević (1982). The data dispersion and discrepancies around $20 \%$ between the different works do not allow establish a clear dependence on temperature.

\section{Experimental set-up and measurements}

All measurements have been made in a pulsed discharge lamp. The experimental set-up (shown in Fig. 1) and the methods have already been described in Gigosos et al. (1994) and Aparicio et al. (1998), so that we will only explain details specific to the present experiment.

The plasmas were created by discharging a capacitor bank of $20 \mu \mathrm{F}$ charged up to $9.5 \mathrm{kV}$, over a cylindrical Pyrex glass lamp (175 mm in length and $19 \mathrm{~mm}$ in interior diameter). During the whole experiment this lamp was working with a continuous flow of neon gas at a rate of $1 \mathrm{~cm}^{3} / \mathrm{min}$, and a pressure of $5 \mathrm{mbar}$, where very small traces of oxygen (actually residual traces) were diluted. In these conditions, self-absorption in OII spectral emission 


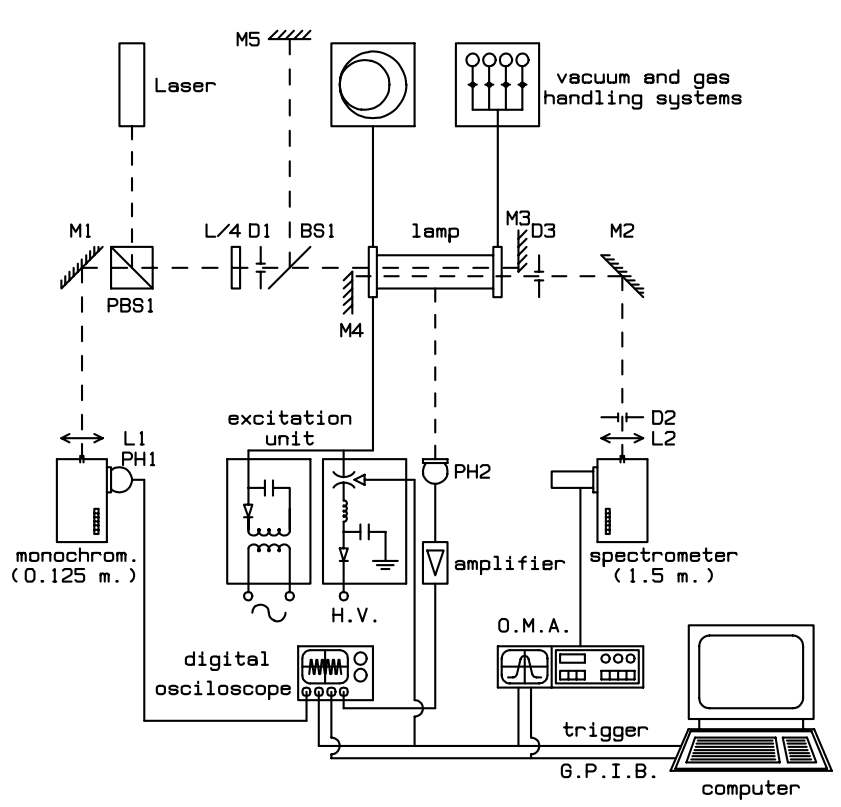

Fig. 1. Experimental set-up

is guaranteed to be completely negligible. The spectral emission lasted for $150 \mu \mathrm{s}$. The gas was pre-ionised in order to have the best discharge reliability. Spectroscopic and interferometric end-on measurements were made simultaneously throughout the plasma life and were taken both $2 \mathrm{~mm}$ off the lamp axis, and from symmetrical positions in relation from the axis. The spatially homogenous plasma and the high cylindrical symmetry of electron density and temperature in our lamp allows this configuration (del Val et al. 1998).

According to Fig. 1, the lamp is placed in one of the arms of a Twyman-Green interferometer illuminated with an argon ion laser (457.9 and $514.0 \mathrm{~nm}$ ). The spectroscopic beam is directed to a Jobin-Yvon spectrometer (1.5 m focal length, 1200 lines/mm holographic grating) equipped with an optical multichannel analyser (OMA). The OMA detector array has 512 channels (EG\&G 1455R-512-HQ). The dispersions in its first, second and third orders of diffraction at $434.0 \mathrm{~nm}$ are 13.08, 5.67 and $2.66 \mathrm{pm} /$ channel respectively. The spectrometer was very carefully calibrated wavelength, as well as intensity (Aparicio et al. 1998; del Val 1997). The OMA was pulsed for $5 \mu \mathrm{s}$ in order to have an acceptable signal-to-noise ratio, a sufficient temporal resolution and to be able to follow the temporal plasma evolution. Mirror M4, placed behind the plasma column, was used to measure the optical depth and to detect possible self-absorption effects on each line profile. This can be performed by comparing the spectra taken with and without the light reflected by this mirror (González et al. 1989). In the experimental plasma conditions, self-absorption was found to be completely negligible.

The experiment consists of end-on measurements of the OII spectra emitted by the plasma in the spectral re-

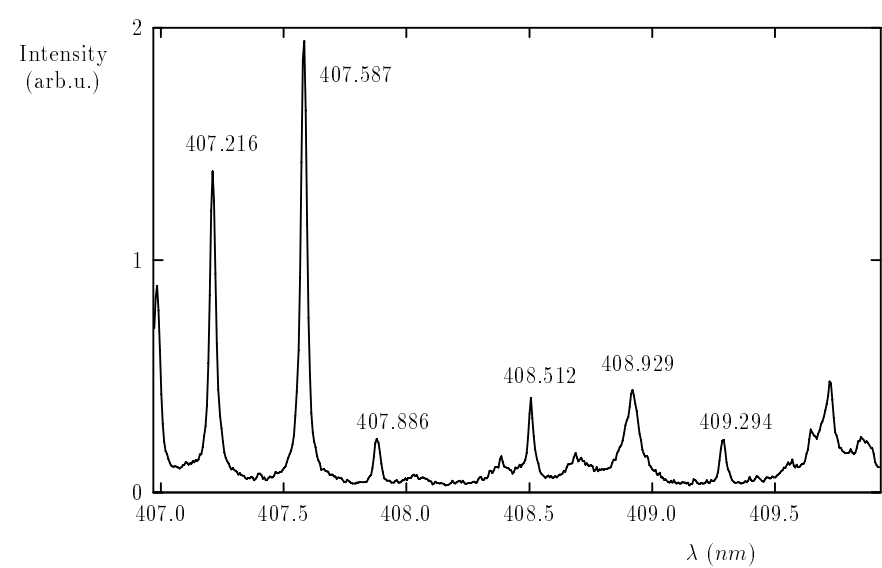

Fig. 2. OMA recorded spectra showing 6 of the OII lines studied in this work. The spectra, registered in second order of diffraction of the spectrometer, correspond to the plasmalife instant $50 \mu \mathrm{s}$

gion $400-470 \mathrm{~nm}$. Each spectral interval was recorded at 13 different instants of the plasma lifetime. For each instant at least three runs were made. An example of a spectrum recorded with 6 OII measured lines at the instant $50 \mu \mathrm{s}$ of the plasma life is shown in Fig. 2. Also, due to the presence of hydrogen impurities in the neon plasma, it was possible to measure the $\mathrm{H}_{\alpha}$ profiles where Stark broadening allowed us to make a spectroscopic determination of the electron density. The OII profile measurements were registered in second order of diffraction of the spectrometer, the most efficient one for the needed spectral resolution.

\section{Calculations and plasma diagnostics}

All the spectra were fitted to sums of Lorentzian functions plus a luminous background with a linear dependence, as explained in Gigosos et al. (1994). Differences between the experimental spectra and the fits were usually lower than $1 \%$. These fitting algorithms allow simultaneous determination of the center, asymmetry, linewidth and area of each profile. While dependent on the chosen line, asymmetries remain below 5\%, which suggests that the ionic contribution to the Stark broadening of OII lines in these plasma conditions is not relevant.

Every fitted spectral line was treated as a Voigt profile. From the whole experimental width $w_{\exp }$ the Lorentzian or Stark component $w_{\mathrm{S}}$ (FWHM) was extracted by using a deconvolution procedure which can be expressed in the following polynomical form:

$$
\begin{aligned}
w_{\mathrm{S}} / w_{\exp }= & 1+0.0029296 x-1.102602 x^{2}+ \\
& +0.0701624 x^{3}+0.0296077 x^{4}
\end{aligned}
$$

where $x=w_{\mathrm{G}} / w_{\exp }$ is the ratio between the Gaussian component and the whole experimental width. This Gaussian component $w_{\mathrm{G}}$ has been calculated from 
Doppler broadening $w_{\mathrm{D}}$ and instrumental width $w_{\mathrm{i}}$ as $w_{\mathrm{G}}=\left(w_{\mathrm{D}}^{2}+w_{\mathrm{i}}^{2}\right)^{1 / 2}$. In this experiment the instrumental profile has a gaussian shape for which FWHM is fundamentally determined by the entrance slit width of the spectrometer, and the measured value results in $w_{\mathrm{i}}=2.6$ OMA channels. For the Doppler width $w_{\mathrm{D}}$ calculation the emitters kinetic temperature was assumed to be close to the OII and NeII excitation temperatures. Taking into account the low gas pressure and the high degree ionisation reached in this experiment, which can be estimated according to Saha law (even greater than 50\%) another broadening mechanisms like neutral pressure or natural width result completely negligible.

Finally, $\mathrm{H}_{\alpha}$ profiles were processed according to the same procedure.

The electron density $N_{\mathrm{e}}$ was determined interferometrically and spectroscopically. In the experiment 156 interferometric registers (1 ms long) were made, the half corresponding to $457.9 \mathrm{~nm}$ and the rest to the $514.0 \mathrm{~nm}$ transition of an argon ion laser. Although these are the most separated available transitions of the laser, in this case, the small difference in wavelength between them does not actually allow double-wavelength interferometry. The differences between the electron density curves obtained from $457.9 \mathrm{~nm}$ one-wavelength interferometry and those corresponding to $514.0 \mathrm{~nm}$ are always lower than $4 \%$ (Fig. 3); hence, we take the mean value of the two curves in all cases.

From each one of the interferograms taken during the experiment, phase evolution was extracted (Aparicio el al. 1998; de la Rosa et al. 1990) by assuming that the plasma column fills the lamp and that refractivity changes are mostly due to free electrons. Both hypotheses have been proved in many works made in this laboratory in recent years (Gigosos et al. 1994; Aparicio et al. 1998; del Val et al. 1997; del Val 1997; de la Rosa et al. 1990). Electron density was also calculated from the hydrogen Balmeralpha Stark width (Fig. 3). This has been calibrated as a function of electron density and temperature in a simulation (Gigosos et al. 1996). In Fig. 3 interferometric and spectroscopic results are compared. As shown in this figure very good agreement between both methods is observed, which seems to confirm the negligible contribution of the bound electrons to refractivity changes and that the effective length of the plasma column is the same as the lamp length. We conclude that $10 \%$ is a good estimation of the electron density uncertainty.

Considering pressure broadening theories and taking into account the electron density and temperature ranges, the OII line profiles are primarily determined by electron impact broadening, so Stark widths are closely related to free electron density and kinetic electron temperature. Moreover, in collision-dominated plasmas like the one generated in this experiment, it is a common hypothesis that excitation temperature and kinetic electron temperature take similar values (Van der Mullen 1990). In this work

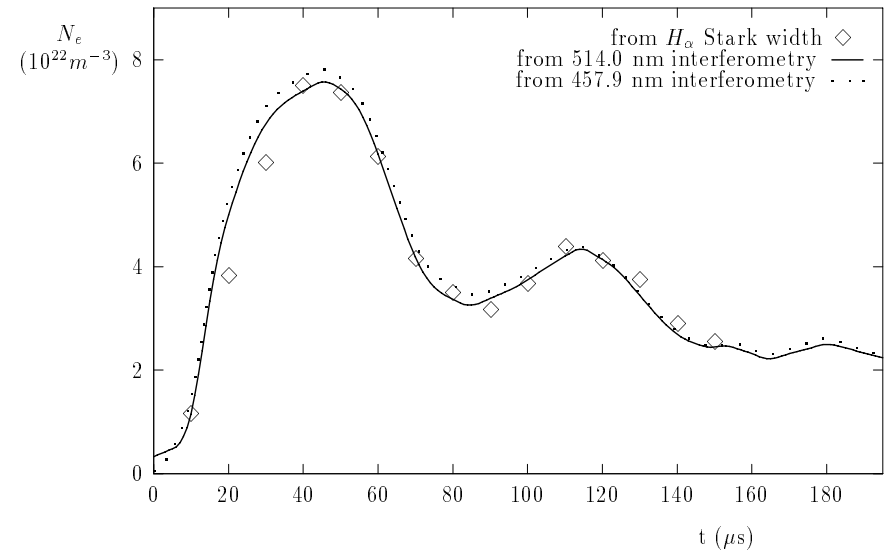

Fig. 3. Electron density evolution measured from interferometry and from $\mathrm{H}_{\alpha}$-Stark broadening

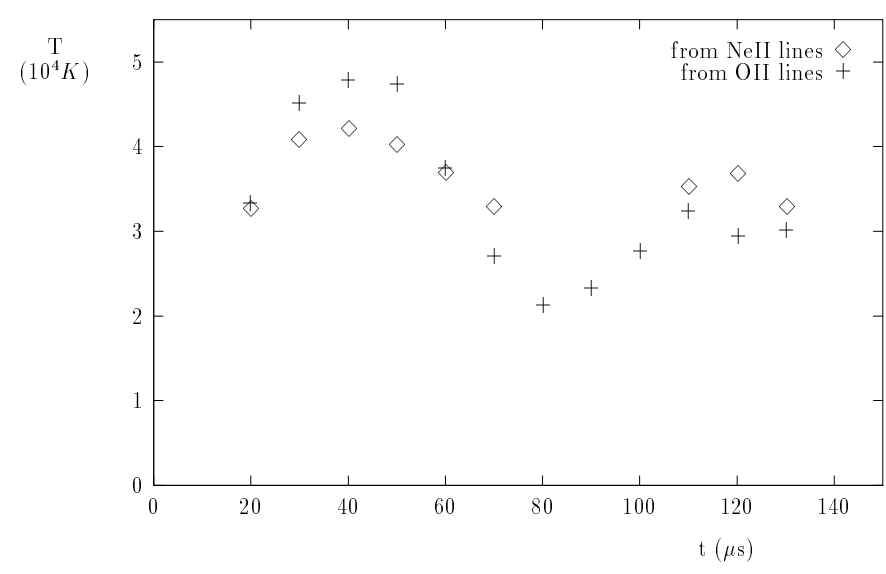

Fig. 4. Electron temperature evolution measured from the Boltzmann-plots of NeII and OII lines

for each instant of the plasma lifetime we determined OII excitation temperature from the slope of a Boltzmannplot calculated with the intensities of a select set of 14 OII lines, whose transition probabilities can be taken from the literature. The same procedure has been followed with 13 selected NeII lines, so that NeII excitation temperature was also determined. Both temperatures are in good agreement, as it is shown in Fig. 4, although the greater one (OII) was taken as electronic temperature, with an estimated uncertainty around $15 \%$.

Due to the narrow features of the OII lines measured in this work we must discuss the influence of the kinetic emitters temperature in the final Stark widths. Although beyond the scope of this work to demonstrate this point, probably a two-temperature (2-T) model for these collision-dominated plasmas could be considered, as already was pointed out by Van der Mullen (1990). If kinetic emitters temperature were lower than kinetic electron temperature, then the Stark width results would increase. Simple calculations prove that, for ion kinetic temperatures of $40000 \mathrm{~K}$ or $20000 \mathrm{~K}$, the resulting Stark widths at $N_{\mathrm{e}}=10^{23} \mathrm{~m}^{-3}$ can differ even up to $30 \%$ for 
Table 1. Different FWHM values (in pm) for the $407.216 \mathrm{~nm}$ OII line emitted at the instant $t=40 \mu \mathrm{s}$. To show the influence of the value taken as emitter kinetic temperature in deconvolution procedure, temperatures of $T=42000 \mathrm{~K}$ and $T=20000 \mathrm{~K}$ are considered. At this instant $N_{\mathrm{e}}=7.4510^{22} \mathrm{~m}^{-3} \cdot w_{\mathrm{D}}=$ Doppler width, $w_{\mathrm{i}}=$ instrumental width, $w_{\mathrm{G}}=$ Gaussian component, $w_{\mathrm{S}}=$ Lorentzian Stark width

\begin{tabular}{llllll}
\hline$T(\mathrm{~K})$ & $w_{\exp }$ & $w_{\mathrm{D}}$ & $w_{\mathrm{i}}$ & $w_{\mathrm{G}}$ & $w_{\mathrm{S}}$ \\
\hline 42000 & 30.15 & 13.30 & 15.11 & 20.13 & 16.20 \\
20000 & 30.15 & 9.18 & 15.11 & 17.68 & 19.31 \\
\hline
\end{tabular}

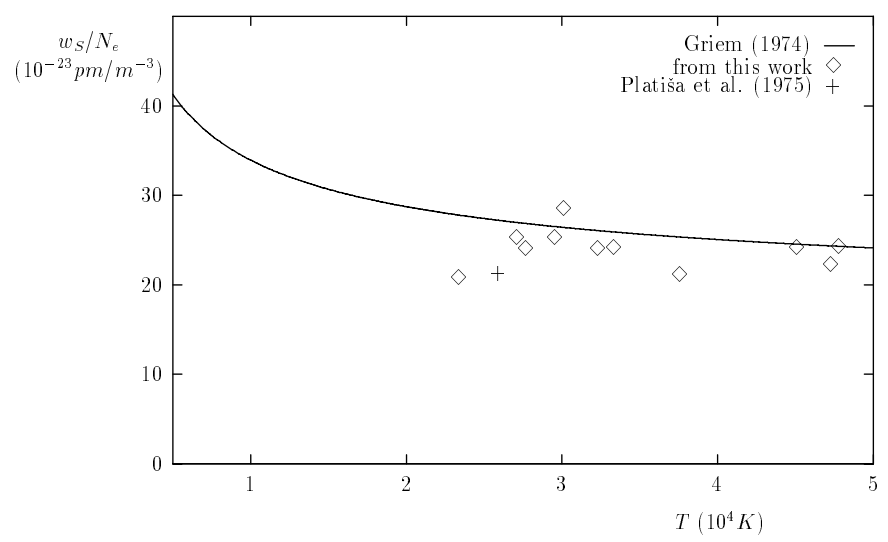

Fig. 5. Example of FWHM Stark widths to $N_{\mathrm{e}}$ ratios versus temperature for the OII line $407.216 \mathrm{~nm}$

the narrowest OII lines. This will be reflected in the uncertainties assigned to these lines. Table 1 shows an example of the full calculations made to obtain the Stark widths $w_{\mathrm{S}}$ from the experimental linewidth $w_{\exp }$ (following the deconvolution procedure already described in Eq. (1)), for the line $407.216 \mathrm{~nm}$ at the plasma life instant $40 \mu \mathrm{s}$. Data obtained considering $42000 \mathrm{~K}$ and $20000 \mathrm{~K}$ as kinetic emitter temperature are compared. As a whole, the best agreement between our OII $w_{\mathrm{S}}$ data and the literature is achieved when considering the kinetic emitters temperature very similar to NeII or OII excitation temperatures.

\section{Results and conclusions}

In order to check possible dependencies of Stark widths on temperature we have plotted the ratios $w_{\mathrm{S}} / N_{\mathrm{e}}$ versus temperature $T$ for each one of the lines. Figure 5 shows an example of these plots for the $407.216 \mathrm{~nm}$ OII line. The available data in the literature are also included. Although in some cases the widths seem to show very slight tendency to decrease with increasing temperature, the scarcity of available data and the inherent uncertainties in the measurements make it difficult to establish any functional relationship, at least in the temperature range considered in this experiment. This behavior is common for every measured line.

In Table 2 the Stark FWHM value $w_{\mathrm{m}}$ is listed for $N_{\mathrm{e}}=10^{23} \mathrm{~m}^{-3}$ obtained from the mean of the ratios
$w_{\mathrm{S}} / N_{\mathrm{e}}$. The electron temperature is assumed to be around $40000 \mathrm{~K}$. The 32 measured OII lines, with their lower and upper transition levels assuming LS-coupling (Striganov \& Sventistskii 1968), are arranged by transition arrays and multiplets according to upper level increasing energy. The number of data and mean is shown for each line, with the corresponding standard statistical error $\sigma$. For comparison, Table 2 shows other experimental available data Platiša et al. (1975); Djeniže et al. (1991, 1998). The accuracy of these data is specified for each case in the Table 2 caption and takes values around $15 \%$ according to the authors. The similar accuracy of the present work takes advantage of a much more extensive set of data and lines. In this way, more reliable uncertainties for each line can be derived from the resulting statistical dispersions. All data have been normalised to $N_{\mathrm{e}}=10^{23} \mathrm{~m}^{-3}$, assuming a linear dependence with $N_{\mathrm{e}}$ and dividing by this value. When available, the ratio of the measured values to theoretical values $w_{\mathrm{m}} / w$ is shown, i.e. semi-classical and semiempirical approaches calculated in Griem (1974); Hey \& Bryan (1977); Hey \& Breger (1980); Dimitrijević (1982) and also results of Purić et al. (1988). These are always for $N_{\mathrm{e}}=10^{23} \mathrm{~m}^{-3}$ and taking, if possible, $T=40000 \mathrm{~K}$.

Our experimental results are usually in good agreement within the same transition arrays and multiplets, as can be seen in Table 2. Also there is good agreement with the theoretical data of Griem and with the measurements of Platiša et al. or Djeniže et al. within 20\% uncertainty ranges. Very reliable Stark data and good experimental profiles of OII emission lines are noticeably difficult to obtain. Also the different plasma experimental conditions, mainly the different temperature ranges, could explain some discrepancies like those corresponding to the $408.929 \mathrm{~nm}$ line. Agreement with Griem, Dimitrijević and Hey \& Breger is good. The work of Hey \& Bryan perhaps slightly underestimates the widths, while the formulae given in the work of Purić et al. (1988) clearly tend to underestimate the widths when applied to $3 \mathrm{~s}-3 \mathrm{p}$ and $3 p-3 d$ transitions of OII specie. Purić et al. (1988) calculated their formula within a semiempirical approach and studied width dependencies on upper level ionization potential working with results of species with different ionization stages, from OII to OV, but mainly OIII.

Taking into account an electron density uncertainty of $10 \%$ we can estimate the average final uncertainty of the Stark widths given in this work around $20 \%$, although with a strong dependence on the line. Perhaps the main error source for the narrowest transitions (often the most intense) is the unknown kinetic emitter temperature in deconvolution procedure. For some other transitions the error comes from weakeness of plasma emission. In spite of the few reliable data which can be obtained for most of the difficult lines, particular uncertainties can be inferred from statistical dispersions. Future high accuracy measurements should extend and complete the lack of OII Stark experimental data. 
Table 2. Experimental Stark widths (FWHM) $w_{\mathrm{m}}$ in pm of OII spectral lines and the ratios to different theoretical values $w_{\mathrm{m}} / w$. All the values are normalised to $N_{\mathrm{e}}=10^{23} \mathrm{~m}^{-3}$. For each line we specify the number of measurements $\mathrm{n}$ taken in this work. [1] Platiša et al. (1975), measures in a pulsed arc, $N_{\mathrm{e}}=0.5210^{23} \mathrm{~m}^{-3}, T_{\mathrm{e}}=25900 \mathrm{~K}$, acc. 16\%. [2] Djeniže et al. (1991), measures in a pulsed arc, $N_{\mathrm{e}}=0.8110^{23} \mathrm{~m}^{-3}, T_{\mathrm{e}}=60000 \mathrm{~K}$, acc. $13 \%$. [3] Djeniže et al. (1998), measures in a pulsed arc, $N_{\mathrm{e}}=2.810^{23} \mathrm{~m}^{-3}$, $T_{\mathrm{e}}=54000 \mathrm{~K}$, acc. 15\%. [4] Griem (1974), theoretical treatment, $N_{\mathrm{e}}=10^{23} \mathrm{~m}^{-3}, T_{\mathrm{e}}=40000 \mathrm{~K}$. [5] Hey \& Bryan (1977), based on Griem semiempirical formulae, $N_{\mathrm{e}}=10^{23} \mathrm{~m}^{-3}, T_{\mathrm{e}}=25900 \mathrm{~K}$. [6] Hey \& Breger (1980), theoretical treatment, method I, $N_{\mathrm{e}}=10^{23} \mathrm{~m}^{-3}, T_{\mathrm{e}}=25900 \mathrm{~K}$. [7] Dimitrijević (1982), semiclassical approximation, $N_{\mathrm{e}}=10^{23} \mathrm{~m}^{-3}$. Interpolated to $T_{\mathrm{e}}=40000 \mathrm{~K}$. [8] Purić et al. (1988), calculated dependences on the upper-level ionization potential, $N_{\mathrm{e}}=10^{23} \mathrm{~m}^{-3}$, $T_{\mathrm{e}}=42500 \mathrm{~K}$, acc. $30 \%$

\begin{tabular}{|c|c|c|c|c|c|c|c|c|c|c|c|c|c|}
\hline $\begin{array}{l}\lambda \\
(\mathrm{nm})\end{array}$ & $\begin{array}{l}\text { Transit. } \\
\text { array }\end{array}$ & Multiplet & $n$ & $w_{\mathrm{m}}$ & $\begin{array}{l}\sigma \\
(\mathrm{pm})\end{array}$ & $\begin{array}{l}w_{\mathrm{m}} \\
{[1]}\end{array}$ & $\begin{array}{l}w_{\mathrm{m}} \\
{[2]}\end{array}$ & $\begin{array}{l}w_{\mathrm{m}} \\
{[3]}\end{array}$ & $\begin{array}{l}w_{\mathrm{m}} / w \\
{[4]}\end{array}$ & $\begin{array}{l}w_{\mathrm{m}} / w \\
{[5]}\end{array}$ & $\begin{array}{l}w_{\mathrm{m}} / w \\
{[6]}\end{array}$ & $\begin{array}{l}w_{\mathrm{m}} / w \\
{[7]}\end{array}$ & $\begin{array}{l}w_{\mathrm{m}} / w \\
{[8]}\end{array}$ \\
\hline 465.084 & $3 s-3 p$ & ${ }^{4} \mathrm{P}-{ }^{4} \mathrm{D}^{\circ}$ & 9 & 22.5 & 3.7 & 23.7 & & & 0.71 & & & 0.73 & 1.37 \\
\hline 463.885 & $3 s-3 p$ & ${ }^{4} \mathrm{P}-{ }^{4} \mathrm{D}^{\circ}$ & 8 & 22.0 & 3.3 & & & 22.9 & 0.70 & & & 0.72 & 1.35 \\
\hline 464.181 & $3 s-3 p$ & ${ }^{4} \mathrm{P}-{ }^{4} \mathrm{D}^{\mathrm{o}}$ & 11 & 22.3 & 6.0 & & & 22.1 & 0.71 & & & 0.73 & 1.36 \\
\hline 464.914 & $3 s-3 p$ & ${ }^{4} \mathrm{P}-{ }^{4} \mathrm{D}^{\mathrm{o}}$ & 12 & 20.9 & 2.0 & 22.9 & & & 0.67 & 1.16 & 0.73 & 0.68 & 1.27 \\
\hline 434.556 & $3 s-3 p$ & ${ }^{4} \mathrm{P}-{ }^{4} \mathrm{P}^{\mathrm{o}}$ & 6 & 25.8 & 4.4 & & & & 1.19 & & & 0.96 & 1.77 \\
\hline 436.689 & $3 s-3 p$ & ${ }^{4} \mathrm{P}-{ }^{4} \mathrm{P}^{\mathrm{o}}$ & 11 & 24.8 & 7.9 & 22.1 & & & 1.13 & & & 0.92 & 1.69 \\
\hline 433.686 & $3 s-3 p$ & ${ }^{4} \mathrm{P}-{ }^{4} \mathrm{P}^{\mathrm{o}}$ & 7 & 20.2 & 5.6 & & & & 0.93 & & & 0.75 & 1.39 \\
\hline 431.714 & $3 s-3 p$ & ${ }^{4} \mathrm{P}-{ }^{4} \mathrm{P}^{\mathrm{o}}$ & 9 & 25.6 & 5.7 & 21.9 & & & 1.18 & 1.62 & 1.06 & 0.95 & 1.78 \\
\hline 434.943 & $3 s-3 p$ & ${ }^{4} \mathrm{P}-{ }^{4} \mathrm{P}^{\mathrm{o}}$ & 9 & 25.3 & 9.0 & & & & 1.16 & & & 0.94 & 1.73 \\
\hline 431.963 & $3 s-3 p$ & ${ }^{4} \mathrm{P}-{ }^{4} \mathrm{P}^{\mathrm{o}}$ & 10 & 26.1 & 6.3 & & & & 1.20 & & & 0.97 & 1.81 \\
\hline 459.617 & $3 \mathrm{~s}^{\prime}-3 \mathrm{p}^{\prime}$ & ${ }^{2} \mathrm{D}-{ }^{2} \mathrm{~F}^{\mathrm{o}}$ & 8 & 27.2 & 4.1 & 24.6 & & $15-13.6^{*}$ & & 1.49 & 0.97 & & \\
\hline 459.097 & $3 \mathrm{~s}^{\prime}-3 \mathrm{p}^{\prime}$ & ${ }^{2} \mathrm{D}-{ }^{2} \mathrm{~F}^{\mathrm{o}}$ & 11 & 22.3 & 7.6 & 25.4 & & & & & & & \\
\hline 435.127 & $3 s^{\prime}-3 p^{\prime}$ & ${ }^{2} \mathrm{D}-{ }^{2} \mathrm{D}^{\mathrm{o}}$ & 9 & 22.5 & 7.1 & & & & & & & & \\
\hline 434.742 & $3 s^{\prime}-3 p^{\prime}$ & ${ }^{2} \mathrm{D}-{ }^{2} \mathrm{D}^{\mathrm{o}}$ & 5 & 31.1 & 8.3 & 21.9 & & & & 1.87 & 1.21 & & \\
\hline 408.512 & $3 p-3 d$ & ${ }^{4} \mathrm{D}^{\mathrm{o}}-{ }^{4} \mathrm{~F}$ & 6 & 25.2 & 7.6 & & & & 0.98 & & & & 2.15 \\
\hline 407.886 & $3 p-3 d$ & ${ }^{4} \mathrm{D}^{\circ}-{ }^{4} \mathrm{~F}$ & 9 & 20.0 & 4.0 & & & & 0.78 & & & & 1.71 \\
\hline 409.294 & $3 p-3 d$ & ${ }^{4} \mathrm{D}^{\mathrm{o}}-{ }^{4} \mathrm{~F}$ & 5 & 21.3 & 2.3 & 23.5 & & & 0.83 & 0.96 & 0.71 & & 1.81 \\
\hline 407.216 & $3 p-3 d$ & ${ }^{4} \mathrm{D}^{\mathrm{o}}-{ }^{4} \mathrm{~F}$ & 11 & 23.9 & 2.0 & 22.7 & & & 0.92 & & & & 2.04 \\
\hline 407.587 & $3 p-3 d$ & ${ }^{4} \mathrm{D}^{\circ}-{ }^{4} \mathrm{~F}$ & 10 & 19.7 & 3.3 & 23.5 & & & 0.76 & & & & 1.68 \\
\hline 415.330 & $3 p-3 d$ & ${ }^{4} \mathrm{P}^{\mathrm{o}}-{ }^{4} \mathrm{P}$ & 7 & 29.3 & 4.9 & 25.6 & & & 1.13 & 1.19 & 0.93 & & 2.38 \\
\hline 415.654 & $3 p-3 d$ & ${ }^{4} \mathrm{P}^{\mathrm{o}}-{ }^{4} \mathrm{P}$ & 6 & 32.9 & 9.8 & & & & 1.26 & & & & 2.67 \\
\hline 413.281 & $3 p-3 d$ & ${ }^{4} \mathrm{P}^{\mathrm{o}}-{ }^{4} \mathrm{P}$ & 9 & 29.9 & 8.3 & 25.4 & & & 1.15 & & & & 2.45 \\
\hline 412.148 & $3 p-3 d$ & ${ }^{4} \mathrm{P}^{\mathrm{o}}-{ }^{4} \mathrm{P}$ & 6 & 24.2 & 8.6 & & & & 0.94 & & & & 2.00 \\
\hline 411.079 & $3 p-3 d$ & ${ }^{4} \mathrm{P}^{\mathrm{o}}-{ }^{4} \mathrm{D}$ & 7 & 21.9 & 5.4 & & & & 0.81 & & & & 1.81 \\
\hline 411.922 & $3 p-3 d$ & ${ }^{4} \mathrm{P}^{\mathrm{O}}-{ }^{4} \mathrm{D}$ & 8 & 24.8 & 6.8 & & & & 0.90 & & & & 2.05 \\
\hline 411.203 & $3 p-3 d$ & ${ }^{4} \mathrm{P}^{\mathrm{o}}-{ }^{2} \mathrm{~F}$ & 5 & 34.3 & 8.0 & & & & & & & & 2.83 \\
\hline 444.821 & $3 \mathrm{p}^{\prime}-3 \mathrm{~d}$ & ${ }^{2} \mathrm{~F}^{\mathrm{o}}-{ }^{2} \mathrm{~F}$ & 10 & 34.3 & 9.0 & & & & & & & & \\
\hline 432.748 & $3 \mathrm{p}^{\prime}-3 \mathrm{~d}^{\prime}$ & ${ }^{2} \mathrm{D}^{\circ}-{ }^{2} \mathrm{D}$ & 6 & 38.2 & 5.1 & & & & & & & & \\
\hline 430.382 & $3 d-4 f$ & ${ }^{4} \mathrm{P}-{ }^{4} \mathrm{D}^{\mathrm{o}}$ & 6 & 118.6 & 23.5 & & 95.6 & & & & & & \\
\hline 408.929 & $3 d-4 f$ & ${ }^{4} \mathrm{~F}-{ }^{4} \mathrm{G}^{\mathrm{o}}$ & 9 & 100.9 & 18.6 & & 66.4 & & & & & & \\
\hline 447.788 & $3 d-4 f$ & ${ }^{2} \mathrm{P}-{ }^{4} \mathrm{G}^{\mathrm{o}}$ & 6 & 127.0 & 20.1 & & & & & & & & \\
\hline 414.609 & $3 \mathrm{p}^{\prime \prime \prime}-3 \mathrm{~d}^{\prime \prime \prime}$ & ${ }^{6} \mathrm{P}-{ }^{6} \mathrm{D}^{\mathrm{o}}$ & 7 & 43.2 & 10.0 & & & & & & & & \\
\hline
\end{tabular}

* Djeniže et al. (1998) take the $459.617 \mathrm{~nm}$ OII line as a doublet (459.600 and $459.617 \mathrm{~nm})$. We found no evidence of this, neither in Striganov \& Sventistskii tables (1968) nor in the DASNIST data base (1990). 
Acknowledgements. We thank Drs. I. de la Rosa, C. Pérez and M.A. Gigosos for their help, S. González for his work in the experimental device, the Dirección General de Investigación Científica y Técnica (Ministerio de Educación y Ciencia) of Spain for its financial support under Contract No. PB-94-0216, and also the Consejería de Educación y Cultura de la Junta de Castilla y León (VA96-96).

\section{References}

Aparicio J.A., Gigosos M.A., González V.R., et al., 1998, J. Phys. B: At. Mol. Opt. Phys. 31, 1029

Davies J.T., Vaaughan J.M., 1963, ApJ 137, 1302

Dimitrijević M.S., 1982, A\&A 112, 251

Djeniže S., Srećković A., Labat J., Platiša M., 1991, Z. Phys. D 21, 295

Djeniže S., Milosavljević V., Srećković A., 1998, J. Quant. Spectrosc. Radiat. Transfer 59, 71

Gigosos M.A., Mar S., Pérez C., de la Rosa I., 1994, Phys. Rev. E 49,1575

Gigosos M.A., Cardeoso V., 1996, J. Phys. B: At. Mol. Opt.
Phys. 29, 4795

González M.A., González V.R., Mar S., 1989, J. Quant. Spectrosc. Radiat. Transfer 42, 247

Griem H.R., 1974, in: Spectral Line Broadening by Plasmas. Academic Press, New York, p. 373

Hey D., Bryan R.J., 1977, J. Quant. Spectrosc. Radiat. Transfer 17, 221

Hey D., Breger P., 1980, J. Quant. Spectrosc. Radiat. Transfer 24,349

Van der Mullen J.A.M., 1990, Phys. Rep. 191, 109

Platiša M., Popović M.V., Konjević N., 1975, A\&A 45, 3255

Purić J., Djeniže S., Srećković A., Platiša M., Labat J., 1988, Phys. Rev. A 37, 498

de la Rosa I., Pérez C., de Frutos A.M., Mar S., 1990, Phys. Rev. A 42, 7389

Striganov A.R., Sventistskii N.S., 1968, in: Tables of Spectral of Neutral and Ionised Atoms. Plenum, New York, p. 146

del Val J.A., 1997, in: Medida de Parametros Atomicos en Plasmas de Neon, Ph.D. Thesis, Univ. Valladolid

del Val J.A., Mar S., Gigosos M.A., de la Rosa I., Pérez C., González V., 1998, Jpn. J. Appl. Phys. 37, 4177 